\title{
ANÁLISE SISTEMÁTICA DAS RESPOSTAS METABÓLICAS DA MICROBIOTA SOB APLICAÇÃO DE HERBICIDAS PRE- EMERGENTES EM SOLOS TROPICAIS
}

Marco Aurélio Pessoa-de-Souza', Liliane Mendes Gonçalves², Marilda da Conceição Ribeiro e Barros $^{3}$, Larissa Gonçalves de Miranda ${ }^{4}$, Nathaza Yohana Amorim Schindel ${ }^{2}$

${ }^{1}$ Doutor em Agronomia. Setor de Solos, Escola de Agronomia, Universidade Federal de Goiás (UFG), Goiânia, Goiás, Brasil ${ }^{2}$ Bacharel em Química. Escola de Ciências Biológicas de Agrárias, Pontifícias Universidade Católica de Goiás (PUC Goiás), Goiânia, Goiás, Brasil

${ }^{3}$ Doutora em Agronomia. Escola de Ciências Biológicas de Agrárias, Pontifícias Universidade Católica de Goiás (PUC Goiás), Goiânia, Goiás, Brasil

${ }^{4}$ Graduandas em Zootecnia. Escola de Ciências Biológicas de Agrárias, Pontifícias Universidade Católica de Goiás (PUC Goiás), Goiânia, Goiás, Brasil

RESUMO: Moléculas como o clomazone, o hexazinone e o imazapic têm ganhado cada vez mais destaque de uso, porém os estudos com foco nos efeitos sobre a microbiota são ainda escassos. Sabe-se que o comportamento de herbicidas no solo é dependente de características físico-químicas das moléculas e do solo, e a biodisponibilidade impacta diretamente na biodiversidade do solo. Portanto, o objetivo deste estudo foi fazer uma revisão sistemática e crítica em torno das similaridades metabólicas do mecanismo de ação dos produtos e das rotas bioquímicas de microrganismos de solo de trabalhos publicados entre 1945 a 2020. Foram criados critérios de seleção que contemplassem estudos que apontassem envidências sobre as similaridades positivas e/ou negativas das moléculas sobre as rotas metabólicas da microbiota do solo. Este estudo demonstrou que a co-participação da molécula no metabolismo microbiano tem relação com a sua disponibilidade e a sensibilidade dos microrganismos do solo.

Palavras-chave: Clomazone, hexazinone, imazapic, metabolismo, disponibilidade. 


\title{
SISTEMATIC ANALYSIS AROUND METABOLICAL RESPONSES OF MICROORGANISMS UNDER PREEMERCING HERBICIDES APPLICATION IN TROPICAL SOILS
}

\begin{abstract}
Molecules as clomazone, hexazinone and imazapic has been priorized to be used in crop field productions, but the studies has not been focused on how they can impact soil microorganisms. It is known that soil herbicide behavior is dependent of physic-chemical characteristics of both soil and molecule, and how availability may directly impact on soil biodiversity. Then, the aim of this study was apply a systematic and critical review around metabolic similarities of the molecules mechanisms of action published from 1945 to 2020. It were organized some selection criteria which were contemplate only studies about positive and/or negative similarities onto soil microorganisms. This present study demonstrated that co-participation of the molecule on microbial metabolism has relation with availability and how sensible are the microorganisms of soil.
\end{abstract}

Keywords: Clomazone, hexazinone, imazapic, metabolism, availability

\section{INTRODUÇÃO}

O uso de pesticidas em escala mundial é crescente, por mais que os dados sejam ainda muito incipientes e desatualizados para os últimos anos. Dentre estes, os herbicidas representam a maior categoria de insumos agrícolas comercializada (FREESE; CROUCH, 2012). Este tipo de tecnologia avança proporcionalmente com organismos geneticamente modificados (OGMs), que também são fonte de muita discussão científica (HILBECK et al., 2015; SÉRALINI et al., 2012). Entretanto, a ciência já conhece o comportamento e efeitos dos pesticidas nos compartimentos ambientais (PRATA, LAVORENTI, 2000).

O Brasil é um dos maiores produtores de alimentos no mundo, e têm uma expectativa positiva de desenvolvimento no setor agrícola, de acordo com OECDFAO (2015). Neste cenário de crescimento, a cana-de-açúcar, uma das maiores commodities do Brasil atualmente, tem muita capacidade de expansão, sobretudo 
no Centro-Oeste (FRANCO et al., 2015) e é uma das maiores consumidoras de herbicidas pré-emergentes (KADWA, 2013),. A rápida expansão das áreas produtivas e sem planejamento trazem problemas ambientais e que podem ser irreversíveis.

Diante dessa problemática, a ciência têm buscado respostas para utilização de agrotóxicos. Arbeli e Fuentes (2010) citam que a IUPAC (International Union of Pure and Applied Chemistry) tem incentivado pesquisas sobre herbicidas, sobretudo a participação dos microrganismos no processo de degradação, na tentativa de compreender os efeitos destes produtos em solos tropicais. Sabe-se que a microbiota do solo faz parte de uma engrenagem dentro do funcionamento dos ciclos biogeoquímicos (BARRIOS, 2007). Sua presença é maior nas primeiras camadas do solo, onde ocorrem as maiores alterações para o preparo do plantio, bem como as aplicações de insumos (CLAPPERTON; MONTANA, 2015). Um solo com alta diversidade microbiana garante 0 bom funcionamento biogeoquímico do sistema, uma vez que eles são utilitários dos nutrientes do solo presentes no meio para sua respiração (WAGG et al., 2014).

Sabe-se que os pesticidas têm a capacidade de alterar a diversidade microbiana no solo (JACOBSEN; HJELMSO, 2014), mais comumente pela pressão de seleção química que os produtos proporcionam ao meio, e em alguns casos até gerando poluição no solo (PRATA; LAVORENTI, 2000). A indústria de insumos agrícola no Brasil é mantida pela forma como o sistema agronômico funciona. Este modelo baseia-se no uso de pesticidas para o controle de matérias biológicos indesejados para ganhos de produtividade. Dentre os complexos agrícolas mais proeminentes para a balança comercial, a cana de açúcar tem contribuído com o aumento do consumo de alguns herbicidas pré-emergentes, como o clomazone, o hexazinone e o imazapic, e consequentemente traz problemas de poluição para o solo, que são totalmente desconhecidos pelos fabricantes dos produtos, bem como para os usuários.

Existem vários estudos que podem contribuir para compreensão dos danos causados pela imperícia no uso de pesticidas no campo, que permeiam entre os parâmetros químicos, biológicos e físicos, estes últimos menos estudados (DUROVIC et al., 2009; GARRETT et al., 2015; GUNASEKARA et al., 2009; 
KODESOVÁ et al., 2011; WANG et al., 2005). Neste sentido, o objetivo deste estudo foi apresentar uma revisão sistemática e crítica em torno das características das moléculas clomazone, hexazinone e imazapic sobre as rotas metabólicas de microrganismos do solo

\section{MATERIAIS E MÉTODOS}

Detalhamento da Sistematização dos Dados

Uma revisão sistemática foi conduzida com base nos em todos estudos realizados sobre o efeito de herbicidas pré-emergentes, especificamente 0 clomazone, hexazinone e imazapic, na microbiota do solo. Diante disso, foram analisados todos os trabalhos publicados em língua portuguesa e inglesa, de 1945 até 2020, disponíveis nas bases de dados do "web of science", "scielo", "scopus", "scifinder", "science direct", "pubmed" e "genome research". Ainda, os critérios estabelecidos para inclusão foram artigos originais de pesquisa em bancada e/ou bancada, de revistas com JRC ou não, qualisada, bem como teses e dissertações, concluídas até dezembro de 2020.

Os termos foram inseridos utilizandos os boleanos "e/ou" ou "or/and", em função da língua utilizada. Para tal, os termos indexados nas bases, em português foram: ("clomazona"e/ou "hexazinona" e/ou "imazapic") e/ou ("pesticida*" e/ou "herbicida*" e/ou "pré-emergente" e/ou "agrotóxico*” e ("microrganismo" e/ou "microorganimo*") e "rota fisiológica" e "degradação" e "metabolismo" e "efeito*" e "comportamento", "impacto" e/ou "DNA". E por sua vez, na língua inglesa: ("clomazone" or/and "hexazinone" or/and "imazapic") or/and ("pesticide" or/and "herbicide" or/and "pre-emergence") and ("microrganism", "physiologic path" and "degradation" and "metabolism" and "effect" and "hehavior", "impact" or/and "DNA".

Os estudos selecionados foram categorizados a partir do resumo, e então submetidos a um teste de avaliação de inclusão sistemática (Tabela 1). Os trabalhos, que não atendessem os critérios estabelecidos foram excluídos, como hipótese de não auxiliarem no estudo. Como exclusão, os trabalhos necessariamente não poderiam ser revisões, ou que não respeitassem os critérios estabelecidos para inclusão. 
Tabela 1: Quadro de teste de relevância para submissão dos resumos avaliados pelas bases de busca Table 1: Relevance test for abstracts evaluated by databases

\begin{tabular}{lll}
\hline \multicolumn{1}{c}{ TESTE DE AVALIAÇÃO DE INCLUSÃO SISTEMÁTICA } & \\
\hline \multicolumn{1}{c}{ Critério de Inclusão } & \multicolumn{1}{c}{ Sim Não } \\
\hline 1. O estudo em questão retrata sobre o comportamento de herbicidas pré- & $\mathrm{X}$ \\
& \\
emergentes em microrganismos do solo? & $\mathrm{X}$ \\
2. Os herbicidas do estudo são pelo menos uma das moléculas de interesse? & \\
& (clomazone, hexazinone e/ou imazapic) & $\mathrm{X}$ \\
3. Os estudos tratam de bactérias e/ou fungos do solo? & $\mathrm{X}$ \\
4. O estudo foi feito em bancada ou a campo? & $\mathrm{X}$ \\
5. O estudo está escrito em língua inglesa ou portuguesa? & $\mathrm{X}$ \\
6. O veículo de publicação é qualisado e apresenta JCR & \\
\hline
\end{tabular}

Após a inclusão dos trabalhos, os artigos foram selecionados e avaliados para discutir sobre a possibilidade dos herbicidas em questão apresentarem algum efeito metabólico sobre a microbiota do solo.

\section{RESULTADOS}

\section{Caracterização e Comportamento dos Herbicidas nos Compartimentos Ambientais.}

Aspectos gerais químicos, bioquímicos e de comportamento do Clomazone.

O clomazone, [2-(2-chlorobenzyl)-4,4-dimethyl-3-isoxazolidinona], (Figura 1) é um herbicida pré-emergente (KRUSE et al., 2001; GUNASEKARA et al., 2009; DARWISH et al., 2013; SHROFF et al., 2014; VAN SCOY; TJEERDEMA, 2014) ou pré-plantio (SHROFF et al., 2014) que pertence ao grupo químico das isoxazolidinonas (DARWISH et al., 2013). Este produto foi desenvolvido no início dos anos 80 (GUNASEKARA et al., 2009; VAN SCOY; TJEERDEMA, 2014) com o nome de Command (GUNASEKARA et al., 2009) e atualmente ele é comercializado com a razão Gamit Star ${ }^{\circledR}$, Gamit 500 EC $^{\circledR}$ e Gamit 360 CS $^{\circledR}$ (SCHREIBER et al., 2013), e apesar de comercialmente ser seletivo (GUNASEKARA et al., 2009; SCHREIBER et al., 2013) no campo ele assume características de amplo espectro (SHROFF et al., 2014) muito utilizado em 
culturas de diferentes portes (ZANELLA et al., 2000; DARWISH et al., 2013; SCHREIBER et al., 2013; SHROFF et al., 2014).

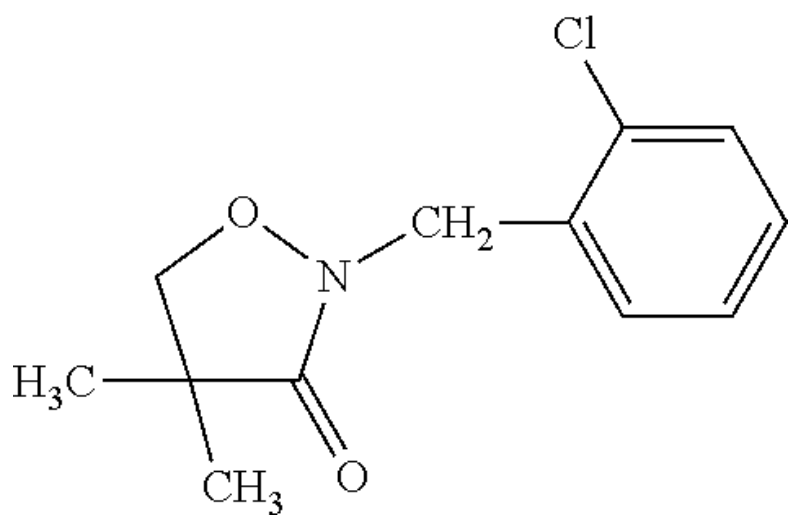

Figura 1: Estrutura molecular do clomazone.

Figure 1: Clomazone Molecular Structure.

É um herbicida muito eficiente, relativamente estável para degradação em luz UV, porém de alta volatilidade, e por isso é muito passível à deriva durante ou após a aplicação, causando danos em plantas não-alvo, que por ventura sejam sensíveis à molécula, como algumas plantas ornamentais, alfafa, pequenos grãos, girassol e hortaliças (SHROFF et al., 2014). É um produto com características físico-química de alta solubilidade em água (1100 mg/L) (ZANELLA et al., 2000; GUNASEKARA et al., 2009), $\mathrm{K}_{\text {oc }}$ de $150 \mathrm{ml} / \mathrm{g}$, e $\mathrm{K}_{d}$ de 0,47 e $5,30 \mathrm{ml} / \mathrm{g}$. (ZANELLA et al., 2000), meia vida de 6 meses e degradação microbiana de 2 a 3 semanas (GUNASEKARA et al., 2009).

Seu principal modo de ação nas plantas é através da inibição da síntese dos carotenóides (GUNASEKARA et al., 2009). Outros modos de ação foram identificados por Ferhatoglu; Barrett (2006), no qual relatam que o clomazone inibe a formação das ligações do cloroplasto com isoprenóides, além de pigmentos fotossintéticos, do tocoferol e de hormônios como a giberelina.

Existem herbicidas que inibem a síntese de carotenoides através da enzima fitoeno desaturase (FD) como o Norflurazon, ou pela inibição da enzima phidroxifenilpiruvato dioxigenase (HPPD), como o Isoxaflutole (KRUSE et al., 2001), já o clomazone apresenta inibição da síntese da geranilgeranil pirofosfatase (GGPP) em células de espinafre, e há fortes indícios da inibição também da isopentenil pirofosfato isomerase (IPP) ou a pernil transferase 
(YASUOR et al., 2010). Ferhatoglu; Barrett (2006) também observaram que em cloroplastos isolados de espinafre um dos metabólitos do clomazone, o 5-keto clomazone, inibiu a produção de deoxixilulose 5 -fosfato (DXP) que é um dos intermediários para a rota do metileritritol 4-fosfato (MEP) (originalmente conhecida por rota do não-mevalonato), que por usa vez participa de uma das rotas da biossíntese de IPP. Neste mesmo estudo os autores confirmaram que o ingrediente ativo clomazone, bem como seu metabólito 5-keto clomazone, inibiram a produção de clorofila e carotenoides.

Vários resultados apontam o impacto do clomazone nos carotenoides e na biossíntese do clorofil que leva ao branqueamento da folhas (fotoxidação da clorofila) (GUNASEKARA et al., 2009; DARWISH et al., 2013; SHROFF et al., 2014). Já anteriormente Ferhatoglu; Barrett (2006) mostraram os indícios que a toxicidade do clomazone não é devido a molécula em si, mas pelos produtos da sua quebra; o 5-ketoclomazone é quebrado para 1-deoxi-Dxilulose-5-fosfato sintase (DXP), a primeira enzima da síntese do IPP na rota do plastídio, alguns anos mais tarde Darwish et al., (2013) também fizeram afirmações semelhantes.

Em termos de comportamento no solo, para o clomazone não é esperado ligações muito fortes, devido seu baixo $\mathrm{K}_{d}$ e sua natureza hidrofílica. Entretanto, tem sido investigado sua interação em diferentes tipos de solo (BROWN; MASIUNAS, 2002; LI et al., 2004; DUROVIC et al., 2009; GUNASEKARA et al., 2009).

Dentre os fenômenos conhecidos do comportamento dos pesticidas, a adsorção é um dos principais mecanismos para a compreensão da ação metabólica da microbiota sobre essas moléculas (DUROVIC et al., 2009). A adsorção é dependente das propriedades do solo, como os teores de matéria orgânica (BROWN; MASIUNAS, 2002; DUROVIC et al., 2009), tipo de argila (BROWN; MASIUNAS, 2002; DUROVIC et al., 2009), capacidade de troca de cátions e pH (LI et al., 2004; DUROVIC et al., 2009).

Em estudos desenvolvidos por Durovic et al. (2009) foi notado que mesmo em solos com teores elevados de matéria orgânica (acima de $8 \%$ ) não há influência para adsorção de clomazone, por outro lado, no solo que apresentou o maior teor de argila, o herbicida ficou mais indisponível. De acordo com os 
autores, este comportamento "inexplicado" pode ser devido aos diferentes usos, aos quais estes solos foram submetido previamente. Resultados similares foram encontrados por Gunasekara et al. (2009), que testaram o comportamento do clomazone no solo por meio de isotermas de adsorção e histerese ${ }^{1}$, e notaram que os ácidos húmicos não influenciam na sua adsorção.

Nos solos da China, Li et al. (2004) encontraram resultados contrários, nos quais as maiores adsorções foram encontradas em solos argilosos com alto teor de matéria orgânica. Loux; Liebl; Slife (1989), muitos anos antes, testaram a adsorção do clomazone em 19 solos diferentes, e apontaram para uma alta correlação da presença de matéria orgânica com a argila, reforçando os resultados encontrados por Li et al. (2004).

Ainda Loux et al. (1989) apontam que as caulinitas e montmorilonitas tem uma alta afinidade por clomazone, e a adsorção aumentam com a capacidade de troca de cátions na argila. Afirmam herbicida não adsorve bem em argilas que estão envolvidas por matéria orgânica, pois sua quantidade de sítios de reação acabam bloqueando os processos de adsorção na superfície das argilas.

Dessa forma, o clomazone possui uma tendência moderada de adsorver em partículas do solo, e por isso apresenta um baixo a moderado potencial de contaminar lençóis freáticos (SHROFF et al., 2014). Não é rapidamente degradado em compartimentos ambientais, como fotólise ou hidrólise, mesmo com diferentes faixas de pH (GUNASEKARA et al., 2009). Ademais, se apresenta como pouco tóxico para mamíferos (LI et al., 2004; VAN SCOY et al., 2014).

\section{Aspectos gerais químicos, bioquímicos e de comportamento do Hexazinone.}

O hexazinone (3-cyclohexyl-6-dimethylamino-1-methyl-1,3,5-triazine-2,4$(1 \mathrm{H}, 3 \mathrm{H})$-dione) (Figura 2) é um herbicida do grupo químico das triazine-dione (triazinadione), e conhecido por ser inibidor da fotossíntese (KENNEDY et al., 2011; RAMOS; YOSHIOKA, 2012; ZHU et al., 2009). Comercialmente é vendido com o nome de $\operatorname{Velpar}^{\circledR} \mathrm{K}$ WG, que é uma mistura de diuron e hexazinone $(46,8 \%$ e $13,2 \%$, respectivamente) (RAMOS; YOSHIOKA, 2012), ou Velpar ${ }^{\circledR} D F$, apenas

\footnotetext{
${ }^{1}$ Fenômeno que ocorre devido a diferença entre as curvas de adsorção e dessorção. 
com hexazinone (75\%) (DuPONT, 2012), absorvido via raiz e translocado para as folhas (RAMSEY; SHIBU, 2004; WANG et al., 2005), e com amplo espectro para controle das áreas (KENNEDY et al., 2011; RAMOS; YOSHIOKA, 2012; ROLANDO et al., 2013; ; Ll et al., 2014).

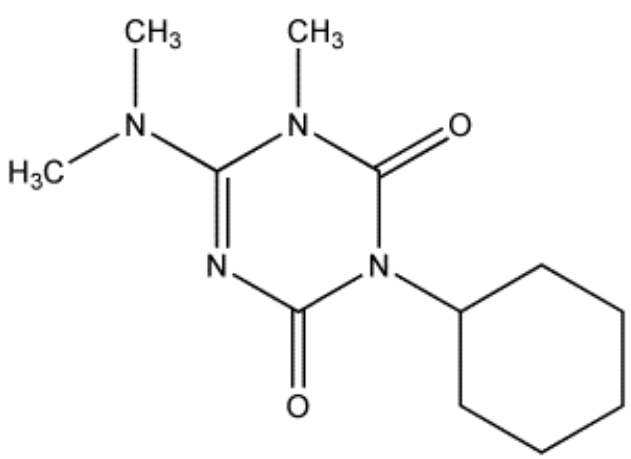

Figura 2: Estrutura molecular do Hexazinone.

Figure 2: Hexazinone Molecular Structure.

O hexazinone enquanto molécula pura, tem solubilidade em água de $33 \mathrm{~g}$ $\mathrm{kg}^{-1}$, Koc de $54 \mathrm{~cm}^{3} \mathrm{~g}^{-1}$, Kow de 15,0 meia vida de 105 dias, $\mathrm{pH}_{\mathrm{a}}$ de 2,2 e é considerado um herbicidas básico e pré-emergente (KODESOVA et al., 2011; NGIGI et al., 2014).

Os herbicidas classificados como inibidores do fotossistema 2 (PS II), se ligam à proteína D1 de forma estequiométrica. Devido a ligação do herbicida, o fluxo de elétrons do PS II é interrompido e a fixação de dióxido de carbono cessa (JABLONKAI, 2011; DAYAN et al., 2015). Dessa forma, os aceptores não ficam disponíveis para receber elétrons para excitação da clorofila, então, radicais livres são formados e desenvolve um quadro de clorose (JABLONKAI, 2011; PETERS et al., 2014). A inibição estequiométrica da D1 como um modo de ação do herbicida não é vantajosa, pois são necessárias altas taxas de aplicação para que se tenha eficiência, uma vez que se trata de uma enzima pouco abundante (PEREZ-JONES et al., 2009; JABLONKAI, 2011).

A enzima D1 participa em etapas de catálise nos tilacóides e tem como atividade precursora, a conversão da proteína D1 inativa em uma forma mais ativa (JABLONKAI, 2011; DAYAN; ZACCARO, 2012), que por sua vez assume a responsabilidade na produção dos pigmentos no cloroplasto, como os carotenóides (DAYAN; ZACCARO, 2012). Apesar do modo de ação do 
hexazinone não ser muito conhecido, Oettmeier et al. (1984), mostraram que em molécula de azido-triazinone, com coordenação moleculares muito semelhante ao hexazinone, ocorreu um forte inibição do transporte de elétrons na fotossíntese (que é oferecido em partes pela enzima D1), e aceptores do PS II, o mesmo já não ocorreu no fotossistema 1 (PS I). Outros estudos reforçam esta hipótese de ação dos herbicidas dos grupos das triazinones (DAYAN; ZACCARO, 2012;).

Em condições de intensa luminosidade a proteína D1 é continuamente exigida e auxilia na manutenção dos complexos PS II, e a eficiência da ação do hexazinone está associada à qualidade e eficiência de atividade desta enzima na planta (JABLONKAI, 2011). Todavia, acredita-se que este mecanismo de ação está associado ao uso de $\operatorname{Velpar}^{\circledR}$, que possui na sua constituição o diuron, isso é reforçado por Moisset et al. (2015), que demonstram que o diuron participa neste fluxo de elétrons com a protease D1 no PS II. Além disso, o diuron demonstra ser mais efetivo no bloqueio da rota metabólica, quando comparado ao hexazinone, que tem seu ataque mais lento na PS II (FLORES et al., 2013).

Em ensaios feitos com Velpar ${ }^{\circledR}$, Metacrate ${ }^{\circledR}$ e Baygon $^{\circledR}$ (CHEN et al., 2014), foi notado que essas moléculas possuem a habilidade de se ligar a um componente chamado luciferase, precursor para bioluminescência de vagalumes, e que participa da produção de ATPs, no inseto. Até o momento este tipo de estudo foi o mais evidente sobre efeito de herbicidas em organismos do Reino Animal.

Nos solos da Nova Zelândia, o hexazinone tem sido reportado como muito móvel, e com meia vida de 79 dias (GARRETT et al., 2015). Os mesmos autores detectaram que em 168 dias, apenas $6 \%$ do hexazinone remanescente de uma aplicação estava presente no solo, o que aponta que este produto tem a capacidade de descer no perfil do solo de forma rápida (em 10 dias), e há uma forte relação com as propriedades físicas do solo. Ainda notaram que a presença de liteira diminui a lixiviação do produto para abaixo de $50 \mathrm{~cm}$ de solo, pois funciona como um compartimento de retenção. Resultados estes apresentado anteriormente por Bicalho et al. (2010), que testaram a distribuição do hexazinone em solos com floresta ripária, que apresentou alta capacidade de mobilidade 
chegando a atingir áreas não-alvo, e compartimentos ambientais indesejados, dessa forma, relacionado com um baixo coeficiente de adsorção $\left(K_{o c}\right)$.

Anteriormente, em outros estudos (CALDERÓN et al., 2004) foi notado que para solos com alto teor de matéria orgânica, em regiões de clima temperado, o Hexazinone ficou menos disponível no solo, entretanto, ainda assim, quando comparado ao Simazine, que pertence ao mesmo grupo químico, apresentou altas reversibilidade de adsorção, o que denota sua baixa capacidade sortiva em solos. Esta baixa capacidade de adsorção na matéria orgânica pode estar associada a uma alta polaridade molecular (alta solubilidade em água) (CELIS et al., 2002).

O hexazinone também não tem habilidade de se adsorver no solo, independente do $\mathrm{pH}$ presente e teor de argila (KODESOVÁ et al., 2011), o que the confere uma alta disponibilidade em termos de biodegradação (WANG et al., 2005), porém apresenta alguma correlação com a capacidade de troca de cátions (CELIS et al., 2002; KODESOVÁ et al., 2011). Em argilominerias que possuem ferro na sua superfície, o hexazinone apresenta maiores capacidade de adsorção (CELIS et al., 2002). Ademais, argilas que tem a capacidade de formar entre si espaço entre as camadas, podem hospedar o hexazinone e controlar sua adsorção por organoargilas (CELIS et al., 2002). Esta capacidade das argilas em funcionar como compartimento ambiental das entrecamadas foi apresentada por vários trabalhos (WANYIKA, 2014).

\section{Aspectos gerais química, bioquímicos e de comportamento do Imazapic.}

O imazapic, 2-[4,5-dihidro - 4 - metil - 4 - (1-metiletil) -5-oxo-1 $\mathrm{H}$ - imidazol 2-il] -5-metil - 3- ácido piridinocarboxílico, (Figura 3), é um herbicida que pode ser usado como pré-emergente (PORFIRI et al., 2015; REIMCHE et al., 2015) e/ou pós-emergência (MARTINI et al., 2013; PORFIRI et al., 2015). Pertencente ao grupo das imidazolinas (REIMCHE et al., 2015), mais especificamente das imidazolinona piridina (KRAEMER et al., 2009), age como inibidor da enzima acetolactato sintase (ALS) (ZHOU et al., 2007) e contém em sua molécula o imidazol, com base em uma segunda estrutura cíclica (KRAEMER et al., 2009). 
Normalmente é utilizado para controle de plantas daninhas em cana de açúcar e arroz (GOULART et al., 2014)

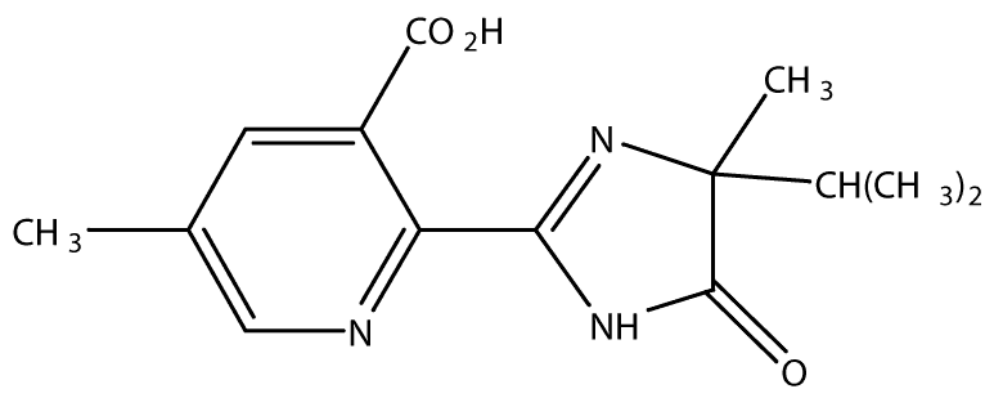

Figura 1: Estrutura molecular do Imazapic.

Figure 1: Imazapic Molecular Structure.

É uma molécula que possui alta solubilidade em água (2200 $\mathrm{mg} \cdot \mathrm{L}^{-1}$ ) (REIMCHE et al., 2015), pKa 3.9 (KRAEMER et al., 2009), Koc de $206 \mathrm{~mL} \cdot \mathrm{g}^{-1}$ (MARTINI et al., 2013), alta mobilidade no perfil do solo, meia vida de 120 dias (REIMCHE et al., 2015).

O primeiro herbicida inibidor de ALS foi comercializado em 1982, com o nome de clorosulfuron, para controle de plantas daninhas de folha larga em cultura de cereais (TRANEL; WRIGHT, 2002), sendo absorvido pelas raízes e folhas e translocado pelo floema e xilema, acumulando-se em pontos de crescimento (KRAEMER et al., 2009).

O ALS, também conhecido por acetohidroxiácido sintase (AHAS) (ZHOU et al., 2007), é a primeira enzima na biossíntese de aminoácidos de cadeia ramificada como a isoleucina, valina e a leucina (TRANEL; WRIGHT, 2002; ZHOU et al., 2007; KRAEMER et al., 2009; ZHOU et al., 2010). Sua inibição conduz a planta a um estádio de estresse por ausência destes aminoácidos, acúmulo de 2cetobutirato, interrupção da síntese de proteínas, perturbações no transporte de fitossintatos (TRANEL; WRIGHT, 2002; ZHOU et al., 2007; KRAEMER et al., 2009).

Já existem relatos de espécies resistente à inibição do ALS (ZHOU et al., 2010; GOULART et al.; 2014), que é causada pela alteração da própria enzima ALS (TRANEL; WRIGHT, 2002), mais especificamente por um ponto de mutação que ocorre no gene da ALS (HUANG et al., 2010; GOULART et al., 2014) e substituição pela planta por alguns aminoácidos. Zhou et al. (2010), apontam que 
essa resistência pode ser devida a alta interação entre a enzima ALS e as moléculas enantiômeras presentes nos produtos.

As imidazolinonas têm uma forte capacidade de adsorção com óxidos de $\mathrm{Fe}$ e Al dos solos devido a carga positiva presente (PORFIRI et al., 2015). Sua adsorção é dependente do tipo de argila, do pH e CTC (REGITANO et al., 2001), entretanto não há qualquer dependência com a presença de MOS (PORFIRI et al., 2015).

Muito antes, Leone et al., 2002, também mostrou que as imidazolinonas tem alta afinidade para adsorção em óxidos de Fe e Al. Isso é devido sua estrutura cristalina que favorece a formação de cargas positivas na superfície e favorece a ligação de ácidos carboxílicos, presente na molécula do imazapic (Figura 3). Esses mesmos resultados são confirmados também por Loux; Reese (1992) e Kraemer et al. (2009), e todos apontam para uma relação direta da adsorção das imidazolinonas com o pH, e a interação dos ácidos húmicos com os complexos sortivos das argilas. Alguns estudos (AICHELE; PENNER, 2005), afirmam que a correlação positiva das imidazolinonas com a matéria orgânica ocorre somente quando a concentração do pesticida no meio é baixa, e o teor de matéria orgânica é elevado.

Os efeitos de sorção das imidazolinonas gira em torno do pH 2.8 e 4.0 (acima e abaixo do pKa deste grupo). A adsorção é em função de do caráter lipofílico da molécula, por isso, em pHs mais baixo as imidazolinonas têm tendência a adsorver mais (NÈGRE et al., 2001). Isso sugere, que para solos do Cerrado Brasileiro, a tendência seja uma alta capacidade de adsorção do imazapic, uma vez que o pH é sempre muito ácido, além da presença de argilas 1:1 e óxidos de Fe e Al (REGITANO et al., 2001; KRAEMER et al., 2009).

As moléculas das imidazolinonas apresentam comportamento anfótero (KRAEMER et al., 2009), possuindo um grupo carboxílico (ácido) e um grupo amino (básico) como grupos funcionais (RAMEZANI et al., 2009), comportando-se como ácidos ou bases fracas, respectivamente (KRAEMER et al., 2009). Chen; Chen et al. (2013), comentam que a adsorção é favorecida em ambientes ácidos, onde o sorbente é imobilizado, e a interação com os ácidos húmico pode ser de 
forma reversível, já para os grupos amina, não são observadas as mesmas relações de interação.

As imidazolinonas possuem a capacidade de formar enantiômeros (RAMEZANI et al., 2009; ZHOU et al. 2010), que possuem radicais do grupo piridina, e apresentam pouca atividade na inibição da ALS (ZHOU et al., 2010), mas exercem efeito diferencial sobre o comportamento no ambiente (KRAEMER et al., 2009), aumentando sua persistência. No entanto, em termos agronômicos, a permanência das imidazolinonas no solo em quantidade suficiente pode comprometer a utilização futura da área com culturas sensíveis ao produto, como alfafa, aveia, milho, pimentão, tomate (KRAEMER et al., 2009).

\section{DISCUSSÃO}

\section{Efeitos dos herbicidas no metabolismo de microrganismos do solo.}

Witshcel et al. (2013) relatam alguns trabalhos que testaram um produto natural, conhecido por fosmidomicina que tem efeitos similares aos causados pelo clomazone. Nestes estudos foram observados a inibição da biossíntese do isoprenóide. Ao mesmo tempo, estes estudos revelaram que algumas plantas e bactérias possuem rotas metabólicas alternativas para o mevalonato, que é um produto chave intermediário, para a produção de isoprenóides e IPP.

Ainda Witshcel et al. (2013) comentam de estudos com análise genômica, nos quais os resultados identificaram uma sequência de sete novos modelos de reação enzimática possíveis, para conversão de gliceraldeído-3-fosfato em IPP.

De acordo com Peters et al. (2014), o clomazone diminui as taxas de crescimento bacteriano. Isso pode ser associado com a alta toxicidade da molécula de clomazone, devido à presença do cloro na molécula (Figura 1). Arjoon; Olaniran; Pillay (2015) reportam bem a cinética de inibição que os compostos com cloro causam, como a diminuição do potencial de biodegradação de alguns compartimentos ambientais.

Outra possível causa é a agressividade do clomazone devido seu mecanismo de ação: inibidor da DXP, que tem o papel chave na biossíntese do isoprenoina, requerido pela tiamina e produção de piridoxal fosfato nos 
procariotos (PETERS et al., 2014), que influencia diretamente a produção de carboidratos e energia metabólica (KEGG, 2015).

Os mesmos autores estudaram o efeito do clomazone aplicado sozinho e em mistura comametrina em concentrações mais altas, neste estudo eles perceberam que a peroxidação de lipídios em Pseudomonas é maior, o que sugere que elas são mais hábeis na degradação destes pesticidas. As Pseudomonas também possuem grande habilidade na degradação de outros herbicidas (PETERS et al., 2014). Liu et al. (1996) ainda no passado, já apontada para uma gama de microrganismos muito grande na degradação de clomazone, cerca de 41 microrganismos foram selecionados para seus estudos em microcosmos, mas 0 Aspergillus niger apresentou 8 tipos metabólitos diferentes para degradação do clomazone, o que indica a sua grande habilidade de degradação deste ingrediente ativo.

Apesar da sua toxicidade, o clomazone tem uma preferência para degradação por meio dos microrganismos, do que fotoquimicamente, como comprovado por Tomco; Tjeerdema (2011).

Ramos; Yoshioka (2012) apontaram de um grupo de microrganismos (EM-4) têm a capacidade de biodegradação em áreas contaminadas (solos e água) por Velpar K, que possui na sua composição o hexazinone.

Anteriormente, Wang et al. (2005) mostrou em seu estudo que Pseudomonas sp e Enterobacter cloacap, em cerca de 10 dias, foram capazes de degradar o hexazinone em meio controlado MSM. Resultados similares foram encontrados por Hunter; Manter et al. (2012), com um proteobactéria (Pseudomonas kuykendallii sp. nov.) isolada de um biorreator para degradação de hexazinone. Ngigi et al. (2014) usaram solos do Kenia, manejados com cana-deaçúcar, para isolamento e identificação de microrganismos com potencial para degradação do hexazinone, e em seus estudos afirmaram que a E. cloacap foi o microrganismo com maior potencial de degradação da molécula.

Para esta molécula é sugerido que a rota de degradação no solo é resultado da perda da cetona, que acompanha o grupo dimetilanino da molécula, por um efeito de hidroxilação (WANG et al., 2005; NGIGI et al., 2014). 
Alguns trabalhos comentam que o hexazinone é capaz de ser biodegradado em aproximadamente 10 dias no solo (WANG et al., 2005), por outro lado, outros estudos apontam que o hexazinone em 50 dias ainda não apresenta grandes avanços no que tange à biodegradação em meio aquoso (MEMIC et al., 2005). Estes resultados levam a inferir que este pesticida em contato com compartimentos que tenham alta quantidade de água, pode apresentar uma maior persistência.

Uma vez que pesticidas como o hexazinone têm uma alta capacidade de off site por lixiviação, tendem a impactar negativamente microrganismos na região da zona vadosa, onde de fato por ocorrer a biodegradação (CLOSE et al., 2010), e onde possui uma microbiota anaeróbia com alta capacidade de degradação (WANG et al., 2009). Essa degradação pode também ser atribuído a microrganismos endógenos devido os baixos níveis e baixa atividade residual que pode durar vários meses (WANG et al., 2005), e que aparentemente não parece ser muito tóxicos para a microbiota.

Ademais, este herbicida tem a capacidade de estimular o crescimento de micorrizas (LAATIKAINEN; HEINONEN-TANSKI, 2002), contrariamente, outros estudos comprovaram que o hexazinone causa problema na formação de ectomicorrizas (BUSSE et al., 2004).

Em ambientes ácidos, onde as imidazolinonas são mais persistentes, os herbicidas são degradados primeiramente pela microbiota (LOUX; REESE, 1992; MOYER et al., 2010). Essa condição de degradação é favorecida pela microbiota do solo, em ambientes tropicais terem um crescimento maior em ambientes onde as condições de acidez são elevadas (ZHANG et al., 2010). Essa atividade microbiana influenciará nas propriedades químicas das imidazolinonas e impedirá sua dissipação na sua forma molecular primária (WANG et al., 2006).

Os pesticidas funcionam como fonte de carbono $(C)$, nitrogênio $(N)$, fósforo (P) e enxofre (S) para os microrganismos do solo, por processos de decomposição, mineralização e imobilização (PERUCCl et al., 2000). O $\mathrm{CO}_{2}$ é uma ferramenta para avaliação dos efeitos dos pesticidas no metabolismo da microbiota (PERUCCl et al., 2000). 
Perucci et al. (2000) identificaram que o imazethapyr, em trinta dias de incubação, apresentou efeitos tóxicos na microbiota do solo, entretanto, em dose dez vezes maior que a dose recomendada a respiração aumentou, mesmo com a diminuição da biomassa microbiana do solo (BMS), o que é indicativo de organismos com alta capacidade de degradação de imidazolinonas. O mesmo ensaio feito com a presença de matéria orgânica, os níveis de BMS se manteram elevados para doses recomendadas e menores para sobredosagens, entretanto as taxas de respiração foram encontradas nas sobredosagens, o que sugere uma elevada pressão de seleção do meio sobre a microbiota. Zhang et al. (2010) encontraram resultados similares para imazethapyr em solos siltosos de regiões temperadas.

Esses resultados reforçam todos os estudos da pouco expressiva participação da matéria orgânica como sítio de adsorção de imidazolinonas (PORFIRI et al., 2015) e como consequência maior disponibilidade para biodegradação (LOUX; REESE, 1992). Em oposição, Moyer et al. (2010), afirmam que a microbiota é estimulada na presença de altas quantidades de matéria orgânica, e dessa forma, a capacidade de degradação microbiana do solo é aumentada, bem como biodegradação de herbicidas.

O mecanismo bioquímico da rota ALS foi também foi identificado em Escherichia coli e Salmonella typhimurium em pelo três tipos chamados de ALS I, II e III, e em Pseudomonas aeruginosa apenas ALS III, e assim como algumas plantas, estes microrganismos desenvolveram resistência e adquiram a capacidade de degradação do pesticida (HUANG et al., 2010). A mesma habilidade foi observada em Arthrobacter crystallopoietes para degradadar as imidazolinonas (WANG et al., 2006). A mutação para resistência das P. aeroginosa foram identificadas no gene il $\mathrm{Vl}$, que codifica um aminoácido mutante no ALS (HUANG et al., 2010). Wang et al. (2006), ainda sugere, que a capacidade de degradação dos microrganismos de solo para as moléculas de imidazolinonas é maior em ambientes anaeróbios.

Estudos moleculares na interação solo-microbiota-herbicidas 
Os efeitos dos pesticidas sobre a estrutura da comunidade microbiana e atividade no solo são motivos para muitos estudos (BUSSE et al., 2004; PERUCCl et al., 2000; PETERS et al., 2014; WITSHCEL et al., 2013; ZHANG et al., 2010). Dada esta condição a medida que ocorreu o desenvolvimento da ciência metodologias se aprimoraram e melhoraram as possibilidades de afirmações dos efeitos dos pesticidas na natureza. O primeiro grande exemplo foi a utilização da quantificação do mRNA mostrando processos de nitrificação, que são muito susceptíveis de alteração para alguns pesticidas (FELD et al., 2015).

$\mathrm{Na}$ última década, uma variedade de ferramentas moleculares têm fomentado os avanços no conhecimento da diversidade e composição das comunidades microbianas no solo (IMFEL; VUILLEUMIER, 2012). Jacobsen \& Hjelmso (2014) trazem em sua publicação um quadro que apresenta maiores detalhes de algumas técnicas que são usadas com objetivos diferentes, como o DGGE, PLFA, PCR em tempo real dentre outras.

Num primeiro momento, a eletroforese em gel de gradiente desnaturante (DGGE) foi uma técnica crucial (SOUZA et al., 2013). O DGGE é uma técnica molecular utilizada para análise de fingerprint rápida da composição da comunidade microbiana, diversidade e dinâmica. $O$ método é rápido e barato, com processamento simultâneo (GREEN et al., 2010). Grandes avanços foram feitos e atualmente é possível fazer extração de DNA em amostras complexas como no solo (SOUZA et al., 2013).

A sensibilidade e o tipo de informação dos dados a nível molecular crescem de forma muito rápida, e é uma ferramenta aplicada à investigações que a priori não poderiam ser resolvida pelos métodos tradicionais. De forma particular, a contribuição e eficiência de alguns filotipos bacterianos no estudo de preditores e bioindicadores (IMFELD; VUILLEUMIER, 2012).

Nos últimos anos, o amplicon $16 \mathrm{~S}$ tratado por pirosequenciamento tem sido muito usado para determinar a diversidade microbiana e estrutura de muitos ecossistemas, e a metagenômica têm se apresentado como a ferramenta mais recente que de fato estuda o potencial metabólico dos microbiomas do solo (CARBONETTO et al., 2014). Dentro deste conceito a metagenômica têm 
crescido, pois estes estudos contribuem para a caracterização taxonômica, e as mudanças na dinâmica funcional.

Por meio de anotação de sequência são criadas categorias funcionais na ordem de genes individuais, ou processos celulares. Basicamente, os genes preditos são tabulados e classificados em categorias funcionais a partir de ordens inferiores (genes individuais) e para ordens superiores (processos celulares). Para entender as características funcionais específicas, as leituras metagenômicas são processadas em um sistema conhecido por Megablast (SAMGWAM et al., 2012), e que podem tomar proporções de estudos taxonômicos como a organização dos grupos por modelos de redes neurais artificiais (da sigla em inglês: ANN) (SANTOS et a., 2014).

Muitos autores se utilizam destas técnicas para responder algumas perguntas, como exemplo as estratégias metabólicas que um solo pode apresentar em um microbioma específico. Neste sentido, Carbonetto et al. (2014) testaram o microbioma de duas condições de adubação, na primeira adubação verde (a partir de restos culturais) e outra adubação mineral convencional. $\mathrm{Na}$ primeira situação os autores notaram que o microbioma apresentou uma tendência de favorecimento de oligotróficos, enquanto que no preparo convencional promoveu o desenvolvimento de copiotróficos, que diminuíram a estabilidade da matéria orgânica do solo, e portanto, aumentou a disponibilidade de nutrientes.

Dessa forma, a compreensão do comportamento metabólico do solo, contribui para de fato conhecer a dinâmica de absorção de nutrientes e eficácia dos itinerários técnicos agrícolas.

\section{CONSIDERAÇÕES FINAIS}

1. Por compartilharem rotas metabólicas similiares, os herbicidas pré-emergente possuem potencial para causar danos nos microrganismos do solo;

2. As Pseudomonas sp. tem notória capacidade de degradação no solo;

3. O impacto negativo dos herbicidas têm relação com as características físicoquímicas do solo e da molécula, e, portanto, a disponibilidade; 
4. As técnicoa de biologia molecular são as que apresentam maior refinamento para demostrar os efeitos sobre os microrganismos no solo;

5. São necessários estudos que evidenciem aspectos técnicos das moléculas, bem como a sua disponibilidade e seu efeito sobre as rotas metabólicas. 


\section{REFERÊNCIAS}

AICHELE, T.M.; PENNER, D. Adsorption, desorption, and degradation of imidazolinones in soil. Weed Technology. v. 19, p. 154-159, 2005. DOI: 10.1614/WT-04-057R

ARBELI, Z.; FUENTES, C.L. Microbial Degradation of Pesticides in Tropical Soils (chapter 12). In: DION, P. (Ed). Soil Biology and Agriculture in the Tropics, Soil Biology, Springer-Verlag Berlin Heidelberg, New York, 2010, 325p.

ARJOON, A.; OLANIRAN, A. O.; PILLAY, B. Kinetics of heavy metal inhibition of 1,2-dichloroethane biodegradation in co-contaminated water. J. Basic Microbiol. v. 55, n. 1, p. 277-284, 2015.

BARRIOS, E. Soil biota, ecosystem services and lad productivity. Ecological Economics. v. 64, n. 1, p. 269-285, 2007.

BICALHO, S.T.T.; LANGENBACH, T.; RODRIGUES, R.R.; CORREIA, F.V.; HAGLER, A.N.; MATALLO, M.B.; LUCHINI, L.C. Herbicide distribution in soils of a riparian forest and neighboring sugar cane field. Geoderma, v. 158, n. 1, p. $392-$ 397, 2010.

BROWN, D.; MASIUNAS, J. Evaluation of herbicides for Pumpkins (Cucurbita spp.). Weed Technology, v. 16, n. 2, p. 282 - 292, 2002. DOI: http://dx.doi.org/10.1614/0890-037X(2002)016[0282:EOHFPC]2.0.CO;2 BUSSE, M.D.; FIDDLER, G.O.; RATCLIFF, A.W. Ectomycorrhizal formation in herbicide-treated soils of differing clay and organic matter content. Water, air, and soil pollution. v. 152, n. 1, p. 23-34, 2004.

CALDERÓN, M.J.; ORTEGA, M.; HERMOSÍN, M.C.; GARCÍA-BAUDÍN, J. CORNEJO, J. Hexazinone and simazine dissipation in forestry field nurseries. Chemosphere, v. 54, n. 1, p. 1-8, 2004.

CARBONETTO, B.; RASCOVAN, N.; ÁlVAREZ, R.; MENTABERRY, A.; VÁZQUEZ, M.P. Structure, composition and metagenomic profile of soil microbiomes associated to agricultural land use and tillage systems in Argentine Pampas. Plos One. v. 9, n. 6, e99949, 2014 DOI: 10.1371/journal.pone.0099949 CELIS, R.; CARMEN HERMOSÍN, M.; CARRIZOSA, J. CORNEJO, J. Inorganic and organic clays as carriers for controlled release of the herbicide hexazinone. J. Agric. Food Chem. v. 50, n. 1, p. 2324-2330, 2002. 
CHEN, C.S.; CHEN, S. Adsorption of pesticidal compounds bearing a single carboxyl functional group and biogenic amines by humic fraction immobilized silica gel. Journal of agricultural and food chemistry. v. 61, p. 3600-3610, 2013. DOI: /10.1021/jf400242c

CHEN, F. LIU, S.S.; DUANA, X.T.; XIAO, Q.F. Predicting the mixture effects of three pesticides by integrating molecular simulation with concentration addition modeling. RSC Advances, n. 4, p. 32256 - 32262, 2014.

CLAPPERTON, J.; MONTANA, R. Pesticide effects on soil biology - part 1. Australina Government - Conservation Agriculture Alliance of Australia \& New Zealand. v. 8, n. 1, p. 1-7, 2015

CLOSE, M.E.; LEE, R.; SARMAH, A.K.; PANG, L.; DANN, R.; MAGESAN, G.N.; WATT, J.P.C.; VICENT, K.W. Pesticide sorption and degradation characteristics in New Zealand soils - a synthesis from seven field trials. New Zealand Journal of Crop and Horticultural Science. v. 36, n. 1, p. 9-30, 2008. DOI: 10.1080/01140670809510217.

CONRAD, R.; NOLL, M.; CLAUS, P.; KLOSE, M.; BASTOS, W.R. e ENRICHPRAST, A.. Stable carbon isotope discrimination and microbiology of methane formation in tropical anoxic lake sediments. Biogeosciences, v. 8, n. 1, p. 795-814, 2011.

DANIEL, R. The metagenomics of soil. Nature. v. 3, p. 470-478, june 2005

DARWISH, M.; LOPEZ-LAURI, F.; SALLANON, H. Study of photosynthesis process in the presence of low concentration of clomazone herbicide in tobacco (Nicotina tabacum). Journal of stress physiology \& Biochemistry, v. 9, n. 1, p. 229245, 2013.

DAYAN, F.E.; ZACCARO, M.L.M. Chlorophyll fluorescence as a marker for herbicide mechanisms of action. Pesticide Biochemistry and Physiology. v. 102, n. 1, p. 189-197, 2012

DAYAN, F.E.; OWENS, D.K.; WATSON, S.B.; ASOLKAR, R.N.; BODDY, L.G. Sarmentine, a natural herbicide from piper species with multiple herbicide mechanisms of action. Frontier in plant science, v. 6, article 222, april 2015. DOI: 10.3389/fpls.2015.00222 
DELMONT, T.O.; ROBE, P.; CECILLON, S.; CLARK, I.M.; CONSTANCIAS, F.; SIMONET, P.; HIRSCH, P.R.; VOGEL, T.M. Accessing the soil metagenome for studies of microbial diversity. Applied and environmental microbiology. v. 77, n. 4, p. 1315-1324, feb. 2011.

DUPONT 2012. Velpar ${ }^{\circledR}$ DF Herbicide. Available at: http://www.dupont.ca/content/dam/dupont/products-and-services/cropprotection/fruit-protection/documents/cp_PSD-59_25225-20121102-sub20123828-Velpar_DF-Label-EN.pdf. Accessed may 14, 2015.

DUROVIC, R.; GAJIÉ-UMILJENDIC, J.; DORDEVIC, T. Effects of organic matter and clay contente in soil on pesticide adsorption processes. Pestic. Phytomed, $v$. 24, n. 1, p. 51-57, 2009.

FAULWETTER, J.L.; GAGNON, V.; SUNDBERG, C.; CHAZAREN, F.; BURR, M.D.; BRISSON, J.; CAMPER, A.K.; STEIN, O.R. Microbial processes influencing performance of treatment wetlands: a review. Ecological Engineering, v. 35, n. 1, p. $987-1004 ; 2009$.

FELD, L. HJELMSO, M.H.; NIELSEN, M.S.; JACOBSEN, A.D.; RONN, R.; EKELUND, F.; KROGH, P.H.; STROBEL, B.W.; JACOBSEN, C.S. Pesticide side effects in an agricultural soil ecosystem as measured by amoA expression quantification and bacterial diversity changes. PLoS One. v. 10, n. 5, e0126080, 2015 DOI: 10.1371/journal.pone.0126080

FERHATOGLU Y.; BARRETT, M. Studies of clomazone mode of action. Pesticide Biochemistry and Physiology, v. 85, n. 1, p. 7-14, 2006.

FLORES, F.; COLLIER, C.J.; MERCURIO, P.; NEGRI, A.P. Phytotoxicity of four photosystem II herbicides to tropical seagrasses. PLoS ONE. v. 8, n. 9, e75798. DOI: 10.1371/journal.pone.0075798.

FRANCO, A.L.; CHERUBIN, M.R.; PAVINATO, P.S.; CERRI, C.E.; SIX, J.; DAVIES, C.A.; CERRI, C.C. Soil carbono, nitrogen and phosphorus changes under sugarcane expansion in Brazil. Sci. Total Environ. v. 15, n. 515-516, p. 3038, 2015.

FREESE, B.; CROUCH, M.L. Comments to USDA APHIS on Draft Environmental Assessment and Draft Plant Pest Risk Assessment for Dow AgroSciences petition (09-349-01p), for determination of nonregulated status of event DAS-68416-4:2,4- 
D- and glufosinate-resistant soybean. Center for Food Safety, Science Comments, 11 sep. 2012.

GARRETT, L.G.; WATT, M.S.; ROLANDO, C.A.; PEARCE, S.H. Environmental fate of terbuthylazine and hexazinone in a New Zealand planted forest Pumice soil. Forest Ecology and Management, v. 337, n. 1, p. 67-76, 2015.

GOULART, I.C.G.R.; BORBA, T.C.O.; MENEZES, V.G.; MEROTTO JUNIOR, A. Distribution of weedy red rice (Oryza sativa) resistant to imidazolinone herbicides and its relationship to rice cultivars and wild Oryza species. Weed Science. v. 62, n. 2, p. 280 - 293, 2014. DOI: 10.1614/WS-D-13-00126.1

GREEN, S.J.; LEIGH, M.B.; NEUFELD, J.D.; Denaturing gradiente gel electrophoresis (DGGE) for microbial community analysis. In.: Handbook of hydrocarbon and lipid microbiology. Ed. Timmis, K.N. Springer Berlin Heidelberg, p. $4137-4158,2010$

GUNASEKARA, A.S.; CRUZ, I.D.P. dela; CURTIS, M.J.; CLAASSEN, V.P.; TJEERDEMA, R.S. The behavior of clomazone in the soil environment. Pest Management Science. v. 65, n. 1, p. 711-716, 2009. DOI 10.1002/ps.1733 HILBECK, A.; BINIMELIS, R.; DEFARGE, N.; STEINBRECHER, R.; SZÉKÁCS, A.; WICKSON, F.; ANTONIOU, M.; BEREANO, P.L.; CLARK, E.A.; HANSEN, M.; NOVOTNY, E.; HEINEMANN, J.; MEYER, H.; SHIVA, V.; WYNNE, B. No scientific consensus on GMO safaty. Environmental Sciences Europe, v. 27, n.4, 2015

HUANG, X.; HE, J.; SUN, X.F.; SUN, J.Q.; LI, Y.F.; SHEN, J.J. LI, S.P. Characterization and molecular mechanism of a naturally occurring metsulfuronmethyl resistant strain of Pseudomonas aeruginosa. World J. Microbiol. Biotechnol. v. 26, n. 1, p. 515-521, 2010.

HUNTER, W.J.; MANTER, D.K. Pseudomonas kuykendallii sp. nov.: A novel Y Proteobacteria isolated from a hexazinone degrading bioreactor. Curr Microbiol v. 65 , n. 1, p. 170-175, 2012. DOI: 10.1007/s00284-012-0141-4.

IMFELD, G.; VUILLEUMIER, S. Measuring the effects of pesticides on bacterial communities in soil: a critical review. European Journal of Soil Biology. v. 49, n. 1, p. 22-30, 2012 
JABLONKAI, I. Molecular mechanism of action of herbicides In.: Hasaneen, M.N.A.E.G.. (Ed). Herbicides - Mechanisms and mode of action. Intech, December 22, p. 4 - 24, 2011

JACOBSEN, C.S.; HJELMSO, M.H. Agricultural soils, pesticides and microbial diversity. Current Opinion in Biotechnology. v. 27, n. 1, p. 15-20, 2014

KADWA, M. An overview of sugarcane supply chain inconsistencies. School of Engineering, University of KwaZulu-Natal, PhD degree. Pietermaritzburg, South Africa, march, 2013, 44p.

KEGG - Kyoto Encyclopedia of Genes and Genomes, Metabolic pathways Aspergillus

niger.In.:http://www.genome.jp/keggbin/show_pathway?org_name=ang\&mapno=0 1100\&mapscale $=0.35 \&$ show_description=hide\&show_module_list $=, 2015$.

KENNEDY, K.J.; BOYD, N.S; NAMS, V.O.; OLSON, A.R. The impacts of fertilizer and hexazinone on sheep sorrel (Rumex acetosella) growth patterns in lowbush blueberry fields. Weed Science, v. 59, n. 3, p. 335-340, 2011.

KOSEDOVA, R.; KOCAREK, M.; KODES, V.; DRÁBEK, O.; KOZAK, J.; HEJTMANKOVÁ, K. Pesticide adsorption in relation to soil properties and soil type distribution in regional scale. Journal of Hazardous Materials. v. 186, n. 1, p. $540-$ 550, 2011.

KRAEMER, A.F.; MARCHESAN, E.; AVILA, L.A.; MACHADO, S.L.O.; GROHS, M. D. Environmental fate of imidazolinone herbicides - a review. Planta Daninha. v. 27, n. 3, p. $629-639,2009$.

KRUSE, N.D.; VIDAL, R.A.; BAUMAN, T.T.; TREZZI, M.M. Sinergismo potencial entre herbicidas inibidores do fotossistema II e da síntese de carotenoides. Ciência Rural, Santa Maria, v. 31, n. 4, p. 569-575, aug, 2001.

LAATIKAINEN, T.; HEINONEN-TANSKI, H. Mycorrhizal growth in pure cultures in the presence of pesticides. Microbiol Res. v. 157, n. 1, p. 127-137, 2002.

LEONE, P.; NĖGRE, M.; GENNARI, M.; BOERO, V.; CELIS, R.; CORNEJO, J. Adsorption of imidazolinone herbicides on smectite-humic acid and smectiteferrihydrite associations. J. Agric. Food Chem. v. 50, p. 291-298, 2002.

LI, Z.; BOYD, N.; MCLEAN, N.; RUTHERFORD, K. Hexazinone resistance in red sorrel (rumex acetosella). Weed Science, v. 62, n. 3, p. 532-537, 2014. 
LI, L.F.; LI, G.; YANG, R.; G, Z.; LIAO, X. Clomazone dissipation, adsorption and translocation in four paddy topsoils. Journal of Environmental Sciences, v. 16, n. 4, p. 678-682, 2004.

LIU, S.Y.; SHOCKEN, M.; ROSAZZA, J.P.N. Microbial transformation of clomazone. J. Agric. Food Chem., v. 44, n. 1, p. 313 - 319, 1996.

LOUX, M.M.; LIEBL, R.A.; SLIFE, F.W. Adsorption of clomazone on soils, sediments, and clays. Weed Science, v. 37, n. 3, p. 440-444, 1989

LOUX, M.M.; REESE, K.D. Effect of soil pH on adsorption and persistence of imazaquin. Weed Science, v. 40, p. 490-496, 1992.

MACRAE, A. The use of $16 \mathrm{~S}$ rDNA methods in soil microbial ecology. Braz. J. Microbiol. v. 31, n. 2, p. 77-82, São Paulo. Apr/ June, 2000

MARTINI, L.F.D.; MEZZOMO, R.F.; AVILA, L.A.; MASSEY, J.H.; MARCHESAN, E.; ZANELLA, R.; PEIXOTO, S.C.; REFATTI, J.P.; CASSOL, G.V.; MARQUES, M. Imazethapyr and imazapic runoff under continuous and intermitente irrigation of paddy rice. Agricultural Water Management. v. 125, p. 26-34, jul, 2013

MEMIC, M.; VRTACNIK, M.; VATRENJAK-VELAGIC, V.; WISSIAK GRM, K.S. Comparative biodegradation studies of pre-emergence broadleaf and grass herbicides in aqueous medium. International Biodeterioration \& Biodegradation. v. 55, n. 1, p. 109-113, 2005.

MOISSET, S.; TIM, K.; MAZEL, F.; MORIN, S.; DELMAS, F.; MAZZELLA, N.; GONZALEZ, P. Genetic and physiological responses of three freshwater diatoms to realistic diuron exposures. Environ Sci Pollit Res, v. 22, n. 1, p. $4046-4055$, 2015. DOI: 10.1007/s11356-014-3523-2

MOYER, J.R.; COEN, G.; DUNN, R.; SMITH, A.M. Effects of landscape position, rainfall, and tillage on residual herbicides. Weed Technology. v. 24, n. 3, p. 361368, 2010. DOI: 10.1614/WT-D-09-00067.1

NĖGRE, M. SCHULTEN, H.R.; GENNARI, M. VINDROLA, D. Interaction of imidazolinone herbicides with soil humic acids. Experimental results and molecular modeling. Journal of environmental science and health part B- Pesticides food contaminants and agricultural wastes. v. 36, n. 2, p. 107-125, 2001. DOI: 10.1081/PFC-100103738 
NGIGI, A.; GETENGA, Z.; BOGA, H.; NDALUT, P. Isolation and identification of hexazinone-degrading bacterium from sugarcane-cultivated soil in Kenya. Bull Environ Contam Toxicol. v. 94, n. 1, p. 364-368, 2014.

OECD/ FAO (Food and Agriculture Organization of the United Nations). Agricultural Outlook 2015-2024. OECD Publishing, Paris, 148p.

OETTMEIER, W.; MASSON, K.; SOLL, H.J.; DRABER, W. Herbicide binding at photosystem II a new azido-triazinone photoaffinity label. Biochimica et Biophysica Acta. v. 767, n. 1, p. 590-595, 1984

PAUL, E.A.; CLARK, F.E. Soil Microbiology and Biochemistry. United Kingdom Edition published by Academic Press Limited, United States of America (USA), 273p.; 1989.

PEREZ-JONES, A.; INTANON, S.; MALLORY-SMITH, C. Molecular analysis of hexazinone-resistant Shepherd's-Purse (Capsella bursapastoris) reveals a novel psbA mutation. Weed Science. v. 57, n. 6, p. 574-578, 2009. DOI: 10.1614/WS09-089.1

PERUCCI, P.; DUMONTET, S.; BUFO, S.A.; MAZZATURA, A.; CASUCCI, C. Effects of organic amendment and herbicide treatment on soil microbial biomass. Biol Fertil Soils. v. 32, n. 1, p. 17-23, 2000.

PETERS, L.P.; CARVALHO, G.; MARTINS, P.F.; DOURADO, M.N.; VILHENA, M.B.; PILEGGI, M.; AZEVEDO, R.A. Differential responses of the antioxidante system of ametryn and clomazone tolerant bacteria. Plos One, v. 9, n. 11, e112271, 2014. DOI: 10.1371/journal.pone.0112271.

PORFIRI, C.; MONTOYA, J.C.; KOSKINEN, W.C.; AZCARATE, M.P. Adsorption and transporto $f$ imazapyr through intact soil columns taken from two soils under two tillage systems. Geoderma. v. 251-252, p. 1-9, aug., 2015

PRATA, F.; LAVORENTI, A. Comportamento de herbicidas no solo: influência da matéria orgânica. Revista Biociências. Taubaté, v. 6, n. 2, p.17-22, jul-dez., 2000

RAJENDHRAN, J.; GUNASEKARAN, P. Strategies for accessing soil metagenome for desired applications. Biotecnology advances. v. 26, n. 1, p. 576590, 2008.

RAMEZANI, M.; SIMPSON, N.; OLIVER, D.; KOOKANA, R.; GILL, G.; PRESTON, C. Improved extraction and clean-up of imidazolinone herbicides from soil 
solutions using diferente solid-phase sorbents. Journal of Chromatography A. v. 1216, p. 5092-5100, 2009

RAMOS, M.A.G.; YOSHIOKA, S.A. Bioremediation of herbicide Velpar $\mathrm{K}^{\circledR}$ in vitro in aqueous solution with application of EM-4 (effective microorganisms). Brazilian archives of biology and technology, v. 55, n 1, p. 145,149, jan, 2012.

RAMSEY, C.; SHIBU, J. Growth, survival and physiological effects of hexazinone and sulfometuron methyl applied overtop of longleaf pine seedlings. Southern Journal of Applied Forestry. v. 28, n. 1, p. 48 - 54 (7), feb 2004.

REGITANO, J.B.; ALLEONI, L.R.F.; TORNISIELO, V.L. Atributos de solos tropicais e a sorção de imazaquin. Sci. Agric. v. 58, n. 4, p. 801-807, 2001. DOI.: 10.1590/S0103-90162001000400023.

REIMCHE, G.B.; MACHADO, S.L.O.; OLIVEIRA, M.A.; ZANELLA, R.; DRESSLER, V.L.; FLORES, E.M.M.; GONÇALVES, F.F.; DONATO, F.F.; NUNES, M.A.G. Imazethapyr and imazapic, bispyribac-sodium and penoxsulam: zooplankton and dissipation in subtropical rice paddy water. Science of the Total Environment. v. 514, p. 68-76, 2015. DOI: 10.1016/j.scitotenv.2015.01.055

SANGWAN, N.; LATA, P.; DWIYEDI, V.; SINGH, A.; NIHARIKA, N.; KAUR, J.; ANAND, S.; MALHOTRA, J.; JINDAL, S.; NIGAM, A.; LAL, D.; DUA, A.; SAXENA, A.; GARG, N.; VERMA, M.; KAUR, K.; MUKHERJEE, U.; GILBERT, J.A.; DOWD, S.E.; RAMAN, R.; KHURANA, P.; KHURANA, J.P.; LAL, RUP. Compartive metagenomic analysis of soil microbial communities across three hexachlorocyclohexane contamination levels. Plos One. v. 7, n. 9, e46219, 2012. DOI: 10.1371/journal.pone.0046219

SANTOS, E.C.; ARMAS, E.D.; CROWLEY, D.; LAMBAIS, M.R. Artificial neural network modeling of microbial community structures in the Atlantic Forest of Brazil. Soil Biology and Biochesmistry. v. 69, n. 1, p. 101-109, 2014.

SCHREIBER, F.; AVILA, L.A.; SCHERNER, A.; MOURA, D.S.; HELGUEIRA, D.B. Plants sensitive to clomazone in vapor phase. Ciência Rural, Santa Maria, v. 43, n. 10, p. 1817-1823, out, 2013.

SÉRALINI, G.E.; CLAIR, E.; MESNAGE, R.; GRESS, S.; DEFARGE, N.; MALATESTA, M.; HENNEQUIN, D.; VENDÔMOIS, J.S. Long term toxicity of a 
roundup herbicide and a roundup-tolerant genetically modified maize. Food and Chemical Toxicology. v. 50, n. 11, p. 4221-4231, 2012

SHROFF, J.R.; SHROFF, V.R.; JADHAY, P.M. BECKER, C., inventors; UPL LIMITED, assignee. Herbicidal formulation. United States Patent Application Publication US 20140200141. Jul 17, 2014

SOUZA, R.C.; CANTÃO, M.E.; VASCONCELOS, A.T.R.; NOGUEIRA, M.A.; HUNGRIA, M. Soil metagenomics reveals diferences under conventional and notillage with crop rotation or succession. Applied Soil Ecology. v. 72, n. 1, p. 49-61, 2013.

TRANEL, P.J.; WRIGHT, T.R. Resistance of weeds to ALS-inhibiting herbicides: what have we learned? Weed Science. v. 50, n. 6, p. 700-712, 2002.

VANCE, E. D.; BOOKES, P. C.; JENKINSON, D. S. An extraction method for measuring soil microbial biomass C. Soil Biology and Biochemistry, Elmsford, 19(6): 703-707, 1987.

VAN SCOY, A.R.; TJEERDEMA, R.S. Enviromental fate and toxicology of clomazone. Rev. Environ. Contam. Toxicol. v. 229, p. 35-49, 2014. DOI: 10.1007/978-3-319-03777-6_3.

WAGG, C.; BENDER, S.F.; WIDMER, F.; VAN DER HEIJDEN, M.G.A. Soil biodiversity and soil community composition determine ecosystem multifunctionality. PNAS. v. 111, n. 14, p. 5266-5270, 2014

WANG, X.; WANG, H.; TAN, C. Degradation and metabolism of hexazionoe by two isolated bacterial strains from soil. Chemosphere. v. 61 , n. 1, p. 1468-1474, 2005

WANG, X.; WANG, H.; FAN, D. Degradation and metabolism of imazapyr in soils under aerobic and anaerobic conditions. International Journal of Environmental Analytical Chemistry. v. $86, \quad$ n. $8, \quad$ p. 541-551, 2006. DOI: 10.1080/03067310500410730.

WANG, H.; XU, S.; TAN, C.; WANG, X. Anaerobic biodegradation of hexazinone in four sediments. Journal of hazardous materials. v. 164, n. 1, p. 806-811, 2009.

WANYIKA, H. Controlled release of agrochemicals intercalated into montmorillonite interlayer space. The Scientific World Journal. v. 2014, n. 656287 , 2014. 
WITSCHEL, M.; RÖHL, F. NIGGEWEG, R.; NEWTON, T. In search of new herbicidal inhibitor of the non-mevalonate pathway. Pest Manag Sci, v. 69, n. 1, 559-563, 2013. DOI: 10.1002/ps.3479.

YASUOR, H.; ZOU, W.; TOLSTIKOV, V.V.; TJEERDEMA, R.S.; FISCHER, A.J. Differential oxidative metabolism and 5-ketoclomazone accumulation are involved in Echinochloa phyllopogon resistance to clomazone ${ }^{[\mathrm{C}][\mathrm{W}][\mathrm{OA}]}$. Plant Physiology, v. 153, p. 319-326, 2010.

ZANELLA, R.; PRIMEL, E.G.; GONÇALVES, F.F.; MARTINS, A.F. Development and validation of a high-performance liquid chromatographic method for the determination of clomazone residues in surface water. Journal of Chromatography A, v. 904, p. 257-262, 2000.

ZHANG, C.; XU, J. LIU, X.; DONG, F.; KONG, Z.; SHENG, Y.. ZHENG, Y. Impact of imazethapyr on the microbial community structure in agricultural soils. Chemosphere. v. 81 , n. 1 , p. $800-806,2010$

ZHOU, Q.; LIU, W.; ZHANG, Y.; LIU, K.K. Action mechanisms of acetolactate synthase-inhibintig herbicides. Pesticides Biochemistry and Physiology. v. 89, n. 2, p. 89-96, oct. 2007

ZHOU, Q.; ZHANG, N.; ZHANG, C.; HUANG, L.; NIU, Y,; ZHANG, Y.; LIU, W. Molecular mechanism of enantioselective inhibition of acetolactate synthase by imazethapyr enantiomers. J. Agric. Food Chem. v. 58 n. 1, n. 4202-4206, 2010. DOI:10.1021/jf9038953 